\title{
Ethik und Organspende
}

\author{
Hartmut Schmidt, Hugo Van Aken
}

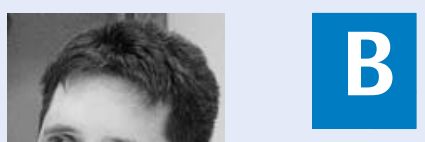

D ereits 1954 transplantierte Joseph Murray in

dessen Zwillingsbruder. Der Patient verstarb 8 Jahre später an den Folgen eines Herz-

infarktes. Seit Einführung des 1. Immunsuppressivums Cyclosporin vor ca. 40 Jahren hat sich die Transplantationsmedizin dynamisch weiterentwickelt. Zurzeit werden routinemäßig Transplantationen der Organe Niere, Leber, Pankreas, Lunge und Herz durchgeführt. Nach anfänglichen Schwierigkeiten setzen sich jetzt auch mehr und mehr Dünndarm- sowie Multiviszeraltransplantationen durch. Je nach Auswahl der Allokationsregeln und der Patienten, die transplantiert werden, können Einjahresüberlebensraten für unterschiedliche Organe bis zu über $90 \%$ erzielt werden. Genetische Erkrankungen wie z.B. bestimmte Formen der hereditären Amyloidose können durch eine Lebertransplantation im Sinne einer Gentherapie die Krankheitsprogression aufhalten. Während früher Patienten mit Hepatitis B nach Lebertransplantation eine eher deutlich eingeschränkte Prognose aufwiesen, ist durch die zusätzliche Einführung von verschiedenen Medikamenten z. B. im Rahmen einer Lebertransplantation eine Heilung der Hepatitis B heutzutage möglich. Die neuerdings eingeführten Immunsuppressiva mit antiproliferativen Eigenschaften werden sowohl in der Transplantationsmedizin zur Immunsuppression als auch in der Onkologie als Chemotherapeutikum eingesetzt. Das wird zukünftig völlig neue therapeutische Optionen in der Betreuung onkologischer Patienten ermöglichen. So können bereits jetzt Patienten mit einem Leberzellkarzinom unter Einhaltung bestimmter Kriterien selbst in fortgeschritteneren Stadien oftmals erfolgreich lebertransplantiert werden. Die Transplantationschirurgie birgt somit die Chance auch zukünftig zunehmend in der Therapie onkologischer Erkrankungen eine Rolle zu spielen. Auch bestehen mittlerweile bereits die ersten Erfahrungen mit sog. CompositeTransplantationen (Transplantationen von Gewebe und Organen). Allein in Deutschland wurden im Jahre 2008 über 2000 Nieren- sowie über 1000 Lebertransplantationen durchgeführt. Insgesamt wurden im Jahre 2008 ungefähr 4000 Organtransplantationen durch hirntote Organspender ermöglicht.

Diese medizinischen Erfolge können jedoch nur gelebt werden, wenn Spenderorgane zur Verfügung stehen. Für die Organspende und die Transplantationsmedizin wurde 1997 in Deutschland erstmalig durch Einführung des Transplantationsgesetzes (TPG) ein juristischer Rahmen festgelegt. Die Lebendspende ist ebenfalls im TPG geregelt. So gilt in Deutschland die erweiterte Zustimmungslösung, sprich, der Spender hat seine Willenserklärung dokumentiert oder in gesetzlicher Reihenfolge dürfen Angehörige im Sinne des mutmaßlichen Willens des Verstorbenen entscheiden. Verschiedene Umfragen in Deutschland zeigen, dass $8-17 \%$ der Bevölkerung einen Organspendeausweis ausgefüllt hat. Auch im Falle eines ausdrücklichen Wunsches für die Organspende ist gesetzlich eine Organspende nur bei ca. $1 \%$ der Verstorbenen umsetzbar. Dies liegt daran, dass nur im Falle eines Hirntods unter kontrollierten Bedingungen auf einer Intensivstation durch Dokumentation von 2 qualifizierten Ärzten unabhängig voneinander ein Hirntod feststellbar und nur in diesem Falle eine Organspende zulässig ist. Die Praxis der Feststellung des Hirntods ( $§ 16$ Absatz 1 Nummer 1 TPG) ist allgemein akzeptiert und hat sich auch in den letzten Jahren bewährt.

Auf den Wartelisten für eine Organtransplantation sterben zurzeit je nach Transplantationszentrum und Organ über 25\% der Patienten. Das heißt, die Mortalität auf einer Transplantationswarteliste ist derzeit höher als das operative Risiko im Rahmen der Transplantation zu sterben, unabhängig von dem zu transplantierenden Organ.

Zur Erhöhung der Organspendezahlen werden verschiedenste Aktivitäten aktuell verfolgt. So gibt es je nach Bundesland Ausführungsgesetze zum TPG, die unter anderem die Einführung von sogenannten Transplantationsbeauftragten festlegen. Der Aufgabenbereich ist teilweise sehr dezidiert festgelegt (Doku- 
mentation interner Verfahrensabläufe der Organspende, Ermittlung von Spenderpotenzial, Unterstützung und Führung von Aufklärungs- und Einwilligungsgesprächen, etc.). Sichergestellt werden sollte, dass diese Tätigkeit als Transplantationsbeauftragter nicht wie viele sonstige ärztliche Tätigkeiten noch zusätzlich durchgeführt wird, sondern hierfür Freiräume in der Ausübung garantiert werden. Auch wird zurzeit über mögliche Sanktionen diskutiert, wenn Krankenhäuser ihrer Meldepflicht nicht nachkommen.

Aber wie sieht die Praxis aus? Ein auf einer Intensivstation eingelieferter Patient mit intracerebraler Blutung und konsekutiver, progredienter, irreversibler Hirnschädigung, mit dilatierten und nicht lichtreagiblen Pupillen, bedarf einer Hirntoddiagnostik. Darf der Intensivmediziner die Angehörigen bezüglich des Willens des Sterbenden ansprechen? Das TPG wird in der Regel so ausgelegt, dass erst im Falle des festgestellten Hirntods sich der Intensivmediziner gemäß dem TPG bei den Angehörigen erkundigen darf. Im Falle einer vorliegenden Patientenverfügung wäre es jedoch sehr nahe liegend das Thema der Organspende zu klären, um die gesamte Tragweite des Willens des Patienten erfassen zu können. Sollte bei diesem Patient während der noch laufenden Hirntoddiagnostik z. B. eine Asystolie auftreten, müssten weitere medizinische Maßnahmen eingestellt werden. Der Patient stirbt am Herztod, eine Organspende - auch wenn es der Patient gewollt hätte - findet nicht statt. Es besteht aber auch die Option, den Hirntod als den endgültigen Tod zu sehen und damit Maßnahmen zu ergreifen, um z.B. durch eine pharmakologische Reanimation, dem Patienten die Herzfunktion aufrechtzuerhalten. Die Feststellung des Hirntods im Verlaufe könnte damit ermöglicht werden, die Frage nach dem festgelegten oder mutmaßlichen Willen für die Organspende wäre dann eruierbar. Es besteht in dieser Behandlungsstrategie jedoch auch die geringe Chance, dass der Patient mit einer irreversiblen hirnorganischen Schädigung mit verbleibender Stammhirnfunktion noch Monate oder Jahre weiterlebt. Letzteres wird vermutlich nicht dem Willen der meisten Menschen in der Bevölkerung entsprechen und widerspräche dem Recht auf würdevolles Sterben (Sterben in Würde, Grundsätze der Bundesärztekammer zur ärztlichen Sterbebegleitung: www.aerzteblatt.de/plus0108). In der Stellungnahme der Bundesärztekammer zum Sterben in Würde ist die ärztliche Verpflichtung aufgeführt, Sterbenden „so zu helfen, dass sie unter menschenwürdigen Bedingungen sterben können“. Weitere Zitate lauten: „Der Arzt kann auch Angehörige des Patienten und diesem nahe stehende Personen informieren, wenn er annehmen darf, dass dies dem Willen des Patienten entspricht.“ und „Bei Patienten, die sich zwar noch nicht im Sterben befinden, aber nach ärztlicher Erkenntnis aller Voraussicht nach in absehbarer Zeit sterben werden, weil die Krankheit weit fortgeschritten ist, kann eine Änderung des Behandlungsziels indiziert sein, wenn lebenserhaltende Maßnahmen Leiden nur verlängern würden und die Änderung des Therapieziels dem Willen des Patienten entspricht“. Diese Zitate bedürfen einer Kommentierung der Bundesärztekammer inwieweit dies auch die Situation der Organspende reflektiert. Den Willen des Sterbenden zu respektieren mit ggf. der Konsequenz einer Änderung des Behandlungsziels bei infauster cerebraler Prognose erfordert, dass der Intensivmediziner auch Zugang zu diesen Informationen hat. Das bedingt die Ermittlung des Willens des Patienten für die Organspende ggf. zu einem früheren Zeitpunkt als das das TPG vorgibt. Die Änderung des Behandlungsziels wäre die Sicherung der Organfunktionen für die Organspende und die damit auch unter Umständen erforderlichen stabilisierenden Maßnahmen infolge des Ausfalls der Hirnfunktionen. Wenn ein Patient sich zu Lebzeiten für die Organspende im Falle des Eintretens eines Hirntods entschieden hat, dann ist ihm auch zu unterstellen, dass er den Organempfängern bestmögliche Organfunktionen ermöglichen möchte.

Die Fortführung der Beatmung sowie eventuell auch die zusätzliche Gabe von Medikamenten, um den Herzkreislauf zu stabilisieren, entsprechen bereits zum Zeitpunkt vor dem Hirntod nicht mehr einer patientenzentrierten Therapie. Der Gesetzgeber hat die realitätsnahe Umstellung von der patientenzentrierten zur organspendezentrierten Therapie nicht berücksichtigt. Es besteht die Befürchtung, dass zu frühzeitig ein möglicher Organspender als hirntot erklärt wird.

Das Vertrauen der Bevölkerung in der Ärzteschaft wird durch die in den Medien geführten Diskussionen zunehmend in Frage gestellt. Dies verschärft auch die Situation in den Entscheidungsprozessen auf der Intensivstation. Ein schriftlich dokumentierter Wille aller Patienten wäre wünschenswert. Aber wie sieht die Situation der Entscheidungsgrundlagen für die Organspende aus? Die Deutsche Stiftung für Organspende (DSO, www.dso.de) hat als Entscheidungsgrundlage für eine Organspende für das Jahr 2007 ermittelt, dass ein schriftlicher Wille in 6,2\%, ein mündlicher Wille in $18,4 \%$ und ein vermuteter Wille in $66,6 \%$ der Fälle vorlag. Die Entscheidungsgrundlage für eine Ablehnung der Organspende war in $0,4 \%$ der schriftliche Wille, in $22,9 \%$ der mündliche Wille und in 47,5\% der Fälle der vermutete Wille. Nur bei 6,6\% lag somit 2007 ein 
schriftlicher Wille vor! Hieraus wird klar ersichtlich, dass auf der Intensivstation in der Regel ausführliche Aufklärungs- und Einwilligungsgespräche für die Organspende geführt werden müssen und zwar zu einem Zeitpunkt, der mit der Überbringung der Todesmitteilung zusammenfällt. In dieser tiefen Trauerarbeit sollte man auf der Intensivstation eine Gesprächssituation schaffen, die es den Angehörigen ermöglicht, sich im Sinne des mutmaßlichen Willens des Verstorbenen zu entscheiden. Bei der Frage, welche Person diese Gespräche führen sollte, ist es sicherlich sinnvoll zu entscheiden, dass der erfahrenste Kollege dafür verantwortlich ist. Koordinatoren der DSO können gemeinschaftlich, aber auch alleine diese Gespräche mit den Angehörigen führen. Ziel sollte sein, dass der betreuende Arzt, der meist bereits Kontakte zu den Angehörigen im Behandlungsprozess auf der Intensivstation hatte, die Bezugsperson für das Gespräch mit den Angehörigen ist.

Die hier geschilderte Situation ist nicht ungewöhnlich. Deshalb bedarf es einer Diskussion in unserer Gesellschaft sowie einer juristischen Neuregelung, um Klarheiten im Prozess Organspende zu schaffen. Es bestehen verschiedene Optionen:

1. Der betreuende Arzt muss eine Einwilligung von den Angehörigen einholen, um die Maßnahmen einer endgültigen Hirntoddiagnostik durchführen zu können. Im Falle des Eintretens und der Bestätigung des Hirntods kann dann der Arzt im Sinne des TPG das Gespräch zur Aufklärung und Einholung der Einwilligung zur Organspende mit den Angehörigen führen.

2. Ärzte dürfen bereits vor Eintreten des Hirntods die Frage der Organspende bei den Angehörigen ansprechen.

3. Man geht von einem presumed consent aus, um Maßnahmen durchführen zu können, die eine endgültige Hirntodfeststellung ermöglichen.

Darüber hinaus muss die Änderung des Behandlungsziels in Reflexion der Organspende diskutiert werden. Letztlich ist auch die Erfahrung und Schulung in der Gesprächsführung der behandelnden Ärzte auf der Intensivstation von besonderer Bedeutung. Die persönliche Interaktion zwischen dem behandelnden Arzt und den Angehörigen steht im Mittelpunkt und ist eine Grundlage, um in dieser schwierigen Trauerphase klare Entscheidungen gemeinschaftlich treffen zu können.

\section{Literatur}

1 Wissenschaftlicher Beirat der Bundesärztekammer. Richtlinien zur Feststellung des Hirntodes. Deutsches Ärzteblatt 1998; 30: A1861 - 1868

2 Siegmund-Schultze N. Transplantationsgesetz: Es fehlt das übergreifende Konzept für die Umsetzung. Deutsches Ärzteblatt 2009; 37: B1530-1533

3 Bericht des IGES-Instituts zur Situation der Transplantationsmedizin in Deutschland 10 Jahre nach Inkrafttreten des Transplantationsgesetzes. Im Internet: http://www.iges.de/publikationen/gutachten/transplantations_studie/e7583/infoboxContent7585/DeutscherBundestagDrucksache1613740_ger.pdf; Stand: 30. 6. 2009

\section{Korrespondenzadressen}

Prof. Dr. Hartmut Schmidt

Transplantationsbeauftragter

Universitätsklinikum Münster

Domagkstr. 3A

48149 Münster

Tel. 0251/83-57770

Fax: 0251/83-57771

E-Mail: hepar@ukmuenster.de

Prof. Dr. Dr. h.c. Hugo Van Aken

Klinik und Poliklinik für Anästhesiologie und operative Intensivmedizin des Universitätsklinikums Münster Albert-Schweitzer-Straße 33

48149 Münster

Tel. 0251-83-47253

Fax: 0251-88704

E-Mail: hva@uni-muenster.de 